\title{
An Infrared Study of Roberts 22
}

\author{
F.J. Molster ${ }^{1}$, L.B.F.M. Waters ${ }^{1,2}$, T. de Jong ${ }^{2,1}$, T. Prusti ${ }^{3}$, A. Zijlstra ${ }^{4}$ and \\ M. Meixner ${ }^{5}$
}

${ }^{1}$ Anton Pannekoek Institute Univ of Amsterdam; ${ }^{2}$ SRON Groningen; ${ }^{3}$ ESA Villafranca;

${ }^{4}$ ESO Garching; ${ }^{5}$ Dept. of Astronomy Univ of Illinois

Roberts 22 (= AFGL 4104) is a peculiar proto-planetary nebula. Its central star has spectral type A2Ie. An OH maser was detected by Manchester et al. (1969), indicating that the shell is oxygen-rich. However Polycyclic Aromatic Hydrocarbons (PAH) features were observed as well (Allen et al., 1982; Cohen et al., 1993), indicating that carbon rich material is also present. In this poster we present new IR data (images and spectra) of this peculiar star and propose an explanation for this curious behaviour.

We present images of Roberts 22 in the J, H, K and N-band. At short wavelengths the nebula is S-shaped and mainly scattered light is visible, indicating a very high dust column density. At longer wavelengths (N-band) the $\mathrm{S}$-shape is less pronounced and the central star becomes visible. The nebula has a size of about 4 arcsec (diameter) in the N-band.

We also present ISO-SWS spectra of this star. The ISO-SWS spectrum of Roberts 22 show PAH emission features at 6.3, 7.8, 8.6, 9.6 and $11.3 \mu \mathrm{m}$, indicating a carbon-rich dust shell. However, in the far-IR oxygen-rich dust appears. These features, also found in other oxygen-rich stars (Waters et al., 1996), may be attributed to the following materials, crystalline olivine (23.5 and $33.7 \mu \mathrm{m})$, clino-pyroxene (32.8 and $40.4 \mu \mathrm{m})$ and crystalline ice $(43.1 \mu \mathrm{m})$. We conclude that Roberts 22 has an oxygen-rich (cool, detached?) and carbon-rich (hot) dust shell. We might look at a transition object, which has recently switched from the oxygen-rich to the carbon-rich state. If the carbon-rich dust comes from the last (and final?) thermal pulse, this event must have happened rather recently, which is supported by the large optical depth to the central source.

Alternatively, the complex geometry and chemistry may point to binarity, and a model similar to that for carbon-rich AGB stars with oxygen-rich envelopes may apply (Barnbaum et al., 1991).

\section{REFERENCES}

Allen D.A. et al, 1982, MNRAS 199, 1017

Barnbaum C., et al., 1991, A\&A 251, 79

Cohen M., et al., 1989, ApJ 341, 246

Manchester R.N. et al. 1969, Astrophys. Lett. 3, 11

Waters L.B.F.M., et al. 1996, A\&A to be published 